\title{
Ultrasound- versus Fluoroscopic-Guided Genicular Nerve Radiofrequency: Which is the Future?
}

\section{To THE EDITOR:}

We intensively read the recent article, "A prospective randomized comparison of the efficacy of ultrasound- vs fluoroscopy-guided genicular nerve block for chronic knee osteoarthritis" written by Kim DH et al (1). In the article, they described "Pain relief, functional improvement, and safety were similar between groups receiving ultrasound- and fluoroscopy-guided genicular nerve blocks. However, considering radiation exposure, ultrasound guidance may be superior to fluoroscopic guidance."

In our pain clinic, after the examination and radiologic evaluations of the patients who applied to the outpatient clinic with the complaint of knee pain, pain treatment interventions are performed with fluoroscopy and ultrasound. In this study, we aimed to determine the effects of the imaging method used during the interventions. We evaluated benefits and risks of ultrasound- versus fluoroscopic-guided genicular nerve radiofrequency in chronic knee pain.

Radiofrequency thermocoagulation was applied to genicular nerves using fluoroscopy or ultrasound in patients who applied to the pain outpatient clinic due to chronic knee pain. After the procedure area was covered under sterile conditions, local anesthetic was applied to the skin and subcutaneous area. Superior medial, inferior medial, and superior lateral genicular nerves were reached by fluoroscopy or ultrasound guidance with a $22-\mathrm{mm}$ radiofrequency needle with a $10-\mathrm{mm}$ active tip. Sensory and motor stimuli were given, the location of the cannula tip was confirmed, and local anesthetic was given after negative aspiration. Conventional radiofrequency thermocoagulation of $80^{\circ} \mathrm{C}$ was performed with each cannula for 90 seconds, and the needle was removed.

The preprocedure Visual Analog Scale (VAS) 1 score was $6.26 \pm 1.00(\min 4.00 ; \max 9.00)$, and the VAS 2 score recorded after the procedure was found to be $3.05 \pm 1.63$. There was a statistically significant difference between VAS 1 and VAS $2(P<0.05)$ scores. It was observed that the severity of pain decreased significantly after the procedure. Entry pain scores (VAS 1) and postprocedure (VAS 2 ) values were significantly reduced in both groups $(P=0.001 ; P<0.05$, respectively). The decrease in pain intensity in the ultrasound group was more pronounced than the fluoroscopy-guided group ( $P=0.002 ; P<0.05$, respectively).

In addition to the treatment effect, evaluating complications are also important in the assessment of a treatment method, but the patient is also exposed to radiation in fluoroscopic guidance procedures and the total cost should also be considered.

The most important factor in the success of these procedures is to perform interventional pain treatment by a specialist who has experience and manage the process by an experienced team. Therefore, this will increase the safety and reduce the complications. Also, these techniques should be performed in specialized centers by trained physicians.

It is very important that these treatments should become safer with improving the experience of physicians and performing the procedures with safer devices.

We think that these scientific discussions will contribute positive effects to the treatment of chronic pain.

\author{
Gozde Dagistan, MD, FIPP \\ Akdeniz University Medical Faculty \\ Department of Anesthesiology \\ Division of Algology \\ 07070 Antalya, Turkey \\ E-mail: g_dagistan@hotmail.com
}

\section{REFERENCES}

1. Kim DH, Lee MS, Lee S, Yoon SH, Shin JW, Choi SS. A prospective random- ized comparison of the efficacy of ultrasound- vs fluoroscopy-guided genicular nerve block for chronic knee osteoarthritis. Pain Physician 2019; 22:139-146. 